\title{
Existence of a metallic phase in a 1D Holstein-Hubbard model at half filling
}

\author{
Phani Murali Krishna ${ }^{a, 1}$, Ashok Chatterjee ${ }^{\text {b,*,2 }}$ \\ a School of Physics, University of Hyderabad, Hyderabad 500046, India \\ b Department of Physics, Bilkent University, 06800 Bilkent, Ankara, Turkey
}

Received 24 January 2007; accepted 22 February 2007

Available online 4 March 2007

\begin{abstract}
The one-dimensional half-filled Holstein-Hubbard model is studied using a series of canonical transformations including phonon coherence effect that partly depends on the electron density and is partly independent and also incorporating the on-site and the nearest-neighbour phonon correlations and the exact Bethe-ansatz solution of Lieb and Wu. It is shown that choosing a better variational phonon state makes the polarons more mobile and widens the intermediate metallic region at the charge-density-wave-spin-density-wave crossover recently predicted by Takada and Chatterjee. The presence of this metallic phase is indeed a favourable situation from the point of view of high temperature superconductivity.
\end{abstract}

(C) 2007 Elsevier B.V. All rights reserved.

PACS: 71.38.-k; 63.20.Kr; 71.10.Fd; 71.10.Pm

It is well known that the Holstein-Hubbard (HH) model provides a very useful and simplest possible framework for the investigation of electron-phonon interaction effects on correlated electrons in narrow-band materials. This model is also important to study the competition between the phonon-induced electron-electron attractive interaction and the direct Coulomb correlation. In the last two decades there have been several investigations [1] on this model and these studies have revealed that the $\mathrm{HH}$ model can capture very many interesting phases of strongly correlated Fermi systems, like charge-density-wave (CDW), spin-densitywave (SDW), superconductivity and so on. In fact, it has been advocated by several authors that the interplay between the phonon-mediated electronic attraction and the direct Coulomb repulsion can be a decisive factor in

\footnotetext{
* Corresponding author. Tel.: +90 312290 2673; fax: +90 3122664127. E-mail address: ashok@fen.bilkent.edu.tr (A. Chatterjee).

${ }^{1}$ Present address: Department of Physics, Bilkent University, 06800 Bilkent, Ankara, Turkey.

${ }^{2}$ On leave from School of Physics, University of Hyderabad, Hyderabad 500046, India.
}

understanding several important and hitherto elusive phenomena like superconductivity in cuprates (see [2,3] for review) and colossal magneto-resistance in manganites [4]. In this context, it is pertinent to mention that one of the mechanisms that has been suggested to explain superconductivity in cuprates is polaronic or bipolaronic $[2,3,5]$. But there has also been a strong opposition to these theories by some researchers according to whom the polaronic/bipolaronic theories require a strong electron-phonon interaction for the formation of polarons and bipolarons which in their view is the greatest bottleneck of these theories because at large electron-phonon coupling a system would normally prefer to settle into a CDW ground state (GS) which is a peierls insulating phase. On the other hand, if the electron-phonon coupling is not too strong to overcome the Coulomb correlation, the system would prefer to be in an SDW GS which is an anti-ferromagnetic Mott insulating phase. Thus, at the very first glance, it may appear that the electron-phonon interaction cannot play any role in high- $T_{\mathrm{c}}$ superconductivity. However, a deeper and more careful look suggests that there could be yet another effect due to the competition between 
the phonon-induced attraction and the Coulomb repulsion leading to some kind of a compromise at the crossover region so much so that the transition from one insulating state to the other may not be direct as normally believed but may go through an intermediate phase and it is this phase which is the subject of our interest. In a recent paper [6], Takada and Chatterjee (TC) have shown for the first time within the framework of one-dimensional (1D) $\mathrm{HH}$ model at half-filling that there may exist an intervening phase at the CDW-SDW crossover region and interestingly enough, this phase is metallic. This theoretical observation is of great importance because such a metallic state, if exists, would be just ideal for high- $T_{\mathrm{c}}$ superconductivity. More recently, Clay and Hardikar [7] have studied the same model by a numerical method based on the density matrix renormalization group technique and confirmed the findings of TC and by calculating the Luttinger liquid correlation exponent $K_{\rho}$ they have further suggested that this phase is indeed superconductive. We believe that this issue being of paramount importance in the current scenario of superconductivity needs a more rigorous analytical investigation and the purpose of the present study is to make an attempt in that direction. The main aim of the present paper is to choose a more improved variational wave function for the phonon subsystem in order to obtain a lower GS energy and then analyse the behaviour of the intermediate metallic phase obtained in [6]. If the width of the metallic phase gets reduced in the improved calculation, then there can be an element of doubt about the veracity of the existence of the metallic phase. On the other hand, if an improved calculation leads to an widening of the metallic phase, then the prediction about the existence of the metallic phase becomes certainly much more stronger.

In [6], TC have investigated the $\mathrm{HH}$ model using a variable-displacement Lang-Firsov (LF) transformation (VDLFT) [8] and a coherent state (CS) as the phonon GS and solved the resulting effective Hubbard model by the exact method of Lieb and $\mathrm{Wu}$ (LW) [9]. However, TC have completely neglected the phonon correlation effect which may play a rather important role as is well known in polaron physics (see [10], for review). In the language of field theory, an electron is the source of phonons, and when an electron emits a phonon it recoils back due to the finite phonon momentum, and while recoiling the electron can emit another phonon, particularly in the case of reasonable electron-phonon coupling, and in that case those two successively emitted virtual phonons will be correlated. This correlation leads to the squeezing of the coherent phonon state and it has been shown by Zheng [11] that it also reduces the Holstein reduction factor considerably and consequently makes the polaron bandwidth larger leading to a higher mobility of the polarons which is more favourable for high- $T_{\mathrm{c}}$ superconductivity. In the present work, we shall include on-site [11] and nearest-neighbour (NN) [12] phonon correlations, which also partially take care of the phonon anharmonicity which has been neglected in the ori- ginal hamiltonian. After including these correlations we shall again bring the phonon system to CS by giving another transformation and finally use the exact Betheansatz solution of LW to obtain the GS energy, the renormalized hopping integral and the relevant phase diagram. Our goal is to show that using a more accurate phonon GS yields results which are more conducive for the phenomenon of superconductivity.

The 1D HH hamiltonian may be written as

$$
\begin{aligned}
\mathscr{H}= & -\sum_{\langle i, j\rangle \sigma} t_{i j} c_{i \sigma}^{\dagger} c_{j \sigma}+U \sum_{i} n_{i \uparrow} n_{i \downarrow}+\omega_{0} \sum_{i} b_{i}^{\dagger} b_{i} \\
& +\sum_{i \sigma} g n_{i \sigma}\left(b_{i}+b_{i}^{\dagger}\right)
\end{aligned}
$$

where $c_{i \sigma}^{\dagger}\left(c_{i \sigma}\right)$ is the creation (annihilation) operator for an electron with spin $\sigma$ at the $i$ th lattice site, $n_{i \sigma}\left(=c_{i \sigma}^{\dagger} c_{i \sigma}\right)$ is the electron number operator, $t_{i j}$ is the bare hopping integral, and $U$ is the on-site Coulomb interaction energy. The notation $\langle i j\rangle$ in the hoping term denotes that the summation over $i$ and $j$ has to be carried over nearest-neighbours only and we shall write $t_{i j}=t . b_{i}^{\dagger}\left(b_{i}\right)$ is the phonon creation (annihilation) operator at the $i$ th site and $\omega_{0}$ is the dispersionless optical phonon frequency. $g$ is the dispersionless electron-phonon interaction strength which may be written as $g=\sqrt{ } \alpha \omega_{0}$, where $\alpha$ is the dimensionless electron-phonon coupling constant.

To obtain a variational solution of (1), we perform a series of canonical transformations, the first being the VDLFT with the generator $R_{1}=\sqrt{ } \alpha \eta \sum_{i \sigma} n_{i \sigma}\left(b_{i}^{\dagger}-b_{i}\right)$, where $\eta$ is a variational parameter. In the conventional LF approach, one chooses $\eta=1$ and obtains the GS energy by averaging the transformed hamiltonian with respect to the zero-phonon state, which however would be a good enough approximation for strong $\alpha$ in the anti-adiabatic limit. In the weak and intermediate coupling region, however, a lower GS energy can be obtained by optimizing $\eta$. Furthermore, VDLFT assumes that the phonon coherence coefficient depends linearly on the electron concentration, $n_{i}$. However, one can also introduce, as shown in [6], an $n_{i}$-independent phonon coherence to lower the energy and that can be achieved by a transformation with a generator $R_{2}=\sum_{i} h_{i}\left(b_{i}^{\dagger}-b_{i}\right)$, where $h_{i}$ is another variational parameter. We shall assume that all sites are identical so that $h_{i}=h \forall i$. The last two transformations together can be expressed in terms of a single generator, $R_{12}=\sum_{i}\left[h_{i}+\right.$ $\left.\sqrt{ } \alpha \eta\left(n_{i}-h_{i} / \sqrt{ } \alpha\right)\right]\left(b_{i}^{\dagger}-b_{i}\right)$. When $\eta=1$, one has the VDLFT and $\eta=0$ gives the $n_{i}$-independent CS transformation (CST). Thus, the two transformations together encompass the entire parameter space of $t$, from anti-adiabatic limit to the adiabatic limit. As already mentioned earlier, we next perform, following Zheng [11], a squeezing transformation with a generator, $R_{3}=\alpha_{\mathrm{s}} \sum_{i}\left(b_{i} b_{i}-b_{i}^{\dagger} b_{i}^{\dagger}\right)$, where $\alpha_{\mathrm{s}}$ is the squeezing parameter to be obtained variationally. This transformation takes care of the phonon correlation at a particular site and thus partially includes the electron recoil effect and secondly, it takes into account through the choice of the phonon state some effects of the anhar- 
monic phonons, i.e., the phonon-phonon interactions which indirectly introduces the finite life-time effects of the phonons and thus incorporates the phonon dynamics in a more realistic way. However, it neglects all inter-site phonon correlations. Following Lo and Sollie [12], we therefore perform a correlated squeezing transformation with a generator, $R_{4}=\frac{1}{2} \sum_{i \neq j} \beta_{i j}\left(b_{i} b_{j}-b_{i}^{\dagger} b_{j}^{\dagger}\right)$, where we choose, for simplicity, $\beta_{i j}=\beta$, when $i$ and $j$ are nearestneighbours and zero otherwise. Obviously the last two transformations spoil the coherence of phonons at the expense of including correlations and introduces some fluctuations. Therefore, in order to deal with these fluctuations, we perform another CST to bring back the phonon coherence with a generator, $R_{5}=\Delta \sum_{i}\left(b_{i}^{\dagger}-b_{i}\right)$, where $\Delta$ is another variational parameter. Averaging the transformed hamiltonian with respect to the zero-phonon state $|0\rangle=\prod_{i}\left|0_{i}\right\rangle$, where the product over $i$ runs over $N$ sites, we then obtain an effective electronic hamiltonian

$$
\begin{aligned}
H_{\text {eff }}= & -J \sum_{i \sigma} n_{i \sigma}-t_{\text {eff }} \sum_{\langle i j\rangle \sigma} c_{i \sigma}^{\dagger} c_{j \sigma}+U_{\text {eff }} \sum_{i} n_{i \uparrow} n_{i \downarrow} \\
& +N \omega_{0}\left[\mathrm{e}^{4 \alpha}\left(\mathrm{e}^{2 \beta}\right)_{00}+\mathrm{e}^{-4 \alpha}\left(\mathrm{e}^{-2 \beta}\right)_{00}-2\right] / 4 \\
& +N \omega_{0} h^{2}+N \omega_{0} M \Delta \mathrm{e}^{2 \alpha_{\mathrm{s}}}\left(2 h+\Delta \mathrm{e}^{2 \alpha_{\mathrm{s}}}\right),
\end{aligned}
$$

with

$$
\begin{aligned}
& J=\omega_{0}\left[\alpha(2-\eta)+2 \sqrt{ } \alpha(1-\eta)\left(h+M \Delta \mathrm{e}^{2 \alpha_{\mathrm{s}}}\right)\right], \\
& U_{\text {eff }}=\left[U-2 \alpha \omega_{0} \eta(2-\eta)\right], \\
& t_{\text {eff }}=t \cdot \exp \left[\alpha \eta^{2} \mathrm{e}^{-4 \alpha_{\mathrm{s}}}\left(\left(\mathrm{e}^{-2 \beta}\right)_{00}-\left(\mathrm{e}^{-2 \beta}\right)_{01}\right)\right], \\
& M=\left(\mathrm{e}^{\beta}\right)_{00}+2\left[\left(\mathrm{e}^{\beta}\right)_{01}+\left(\mathrm{e}^{\beta}\right)_{02}+\left(\mathrm{e}^{\beta}\right)_{03}+\cdots\right], \\
& \left(\mathrm{e}^{ \pm 2 \beta}\right)_{0 n}=\sum_{m=0,1,2 \ldots}( \pm 1)^{n} \frac{(2 \beta)^{2 m+n}}{m !(m+n) !} .
\end{aligned}
$$

Eq. (2) is an effective 1D Hubbard hamiltonian which has an exact solution at half-filling for $U_{\text {eff }}>0$ [9]. In [6] TC have extended the LW solution also for the negative values of $U_{\text {eff. }}$ The GS energy per site is thus obtained for all values of $U$ as

$$
\begin{aligned}
\varepsilon_{0}= & -J+N \omega_{0}\left[h^{2}+\left(\left(\mathrm{e}^{2 \beta}\right)_{00} \cosh 4 \alpha-1\right) / 2\right] \\
& +\left[\left(U_{\text {eff }}-\left|U_{\text {eff }}\right|\right) / 4\right]+N \Delta \omega_{0} M \mathrm{e}^{2 \alpha_{\mathrm{s}}}\left(2 h+\Delta \mathrm{e}^{2 \alpha_{\mathrm{s}}}\right) \\
& -4 t_{\text {eff }} \int_{0}^{\infty} \mathrm{d} y \frac{J_{0}(y) J_{1}(y)}{y\left[1+\exp \left(y\left|U_{\text {eff }}\right| / 2 t_{\text {eff }}\right)\right]} .
\end{aligned}
$$

The GS energy is now minimized with respect to five variational parameters $\eta, h, \alpha_{\mathrm{s}}, \beta$, and $\Delta$ and the results are shown in Fig. 1. To see the efficacy of the present method we also plot the TC results. It is evident that the energy results are only marginally better. However, as we shall show, even this marginal improvement in the energy can have substantial effect on the polaron mobility and the phase diagram. One may note that the energy results again show some kind of rounding off suggesting the existence of an intermediate phase.

In Fig. 2, we have plotted $t_{\text {eff }}$ and its derivative. For sufficiently small $U, U_{\text {eff }}<0$ and as the figure shows, $t_{\mathrm{eff}}$ is also

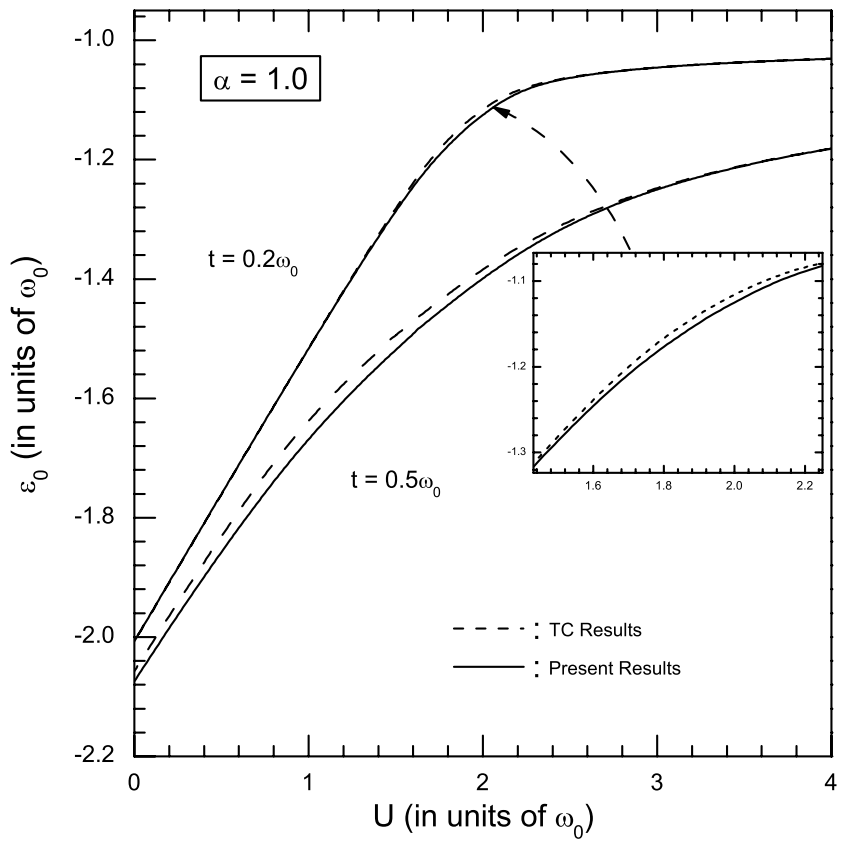

Fig. 1. $\varepsilon_{0}$ as a function of $U$ for $\alpha=1$ and two values of $t$. The results of [6] are shown for comparison.

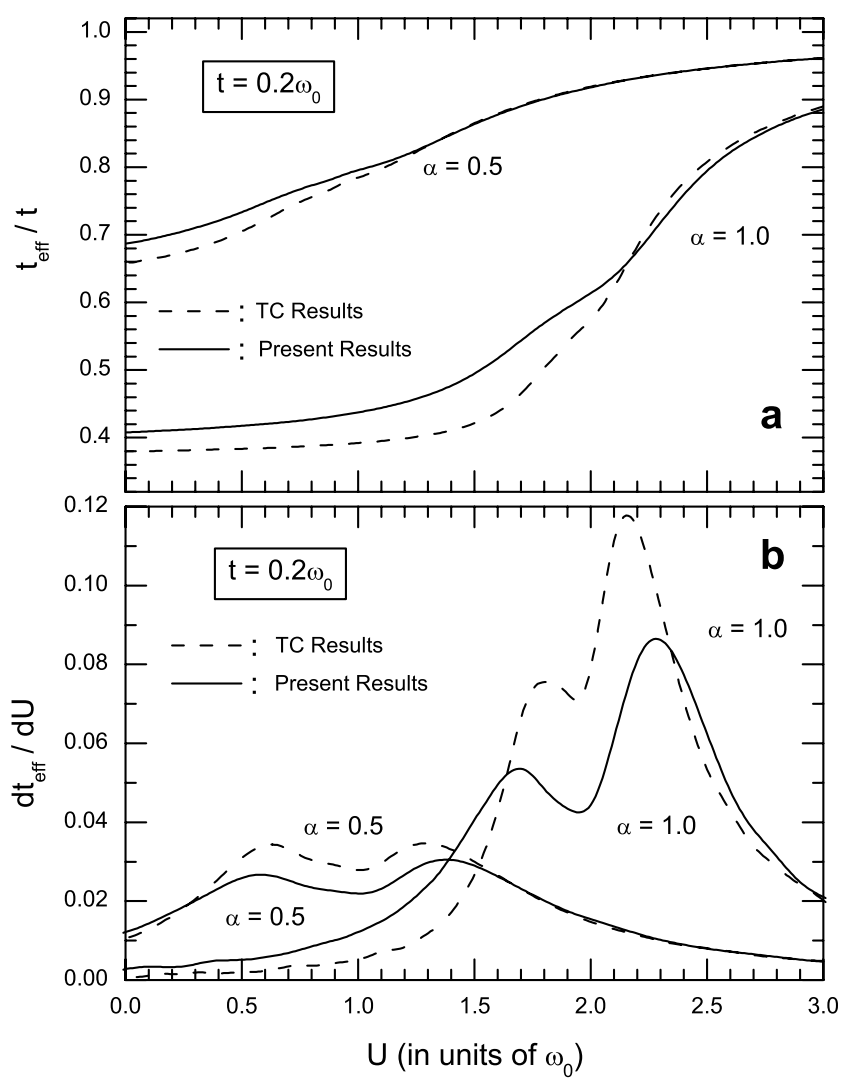

Fig. 2. $t_{\mathrm{eff}}$ and its derivative as a function of $U$ for two values of $\alpha$ and for $t=0.02 \omega_{0}$. The TC results are shown for comparison.

very small. As a result, massive bipolarons form and the band becomes narrow. This is the insulating CDW state or the Peierls state. When $U$ becomes large, one gets the 
usual Hubbard model and then the GS is the SDW state giving an antiferromagnetic Mott insulator. But the important point to note here is that the present method leads to an enhancement in the polaron mobility as compared with the TC results save for a small region of $U$ where the two methods almost agree. As in [6], between the two phases, again some curious features appear and to unravel these we plot in Fig. 2b, d $t_{\text {eff }} / \mathrm{d} U$ as a function of $U$ and now distinct peaks appear on either side of the crossover point defined by $U_{\text {eff }}=0$. The region in between the transition points satisfies the criterion $4 t_{\text {eff }} / U_{\text {eff }} \gtrsim 1$, typical of a metallic state. Now the peaks are a little wider apart as compared to the TC results. In Fig. 3, we draw the phase diagram in the parameter space $(\alpha, U)$, the boundaries of which are determined from the peak positions of the $\mathrm{d} t_{\mathrm{eff}} /$ $\mathrm{d} U$ curve of Fig. 2. Comparison with the TC results again clearly shows that the present method predicts a wider metallic phase at the crossover region.

Next, we calculate the local spin moment $L_{0}$ given by $L_{0}=\sum_{i}\left\langle S_{i}^{2}\right\rangle / N=0.75-1.5 \sum_{i}\left\langle n_{i \uparrow} n_{i \downarrow}\right\rangle / N$ which can be written as $[6], L_{0}=0.75-1.5\left[\mathrm{~d} \varepsilon_{0} / \mathrm{d} U\right]$. In Fig. 4 , we show the contour plots of $L_{0}$ in the $(\alpha, \beta)$ plane.

It was mentioned in [6] that for a completely uncorrelated electron gas $L_{0}=0.375$ which is indeed the value that we see in the intermediate phase. This is another evidence confirming the existence of an intervening metallic phase at the CDW-SDW crossover region. Comparison with the corresponding contour plots of [6] shows that the

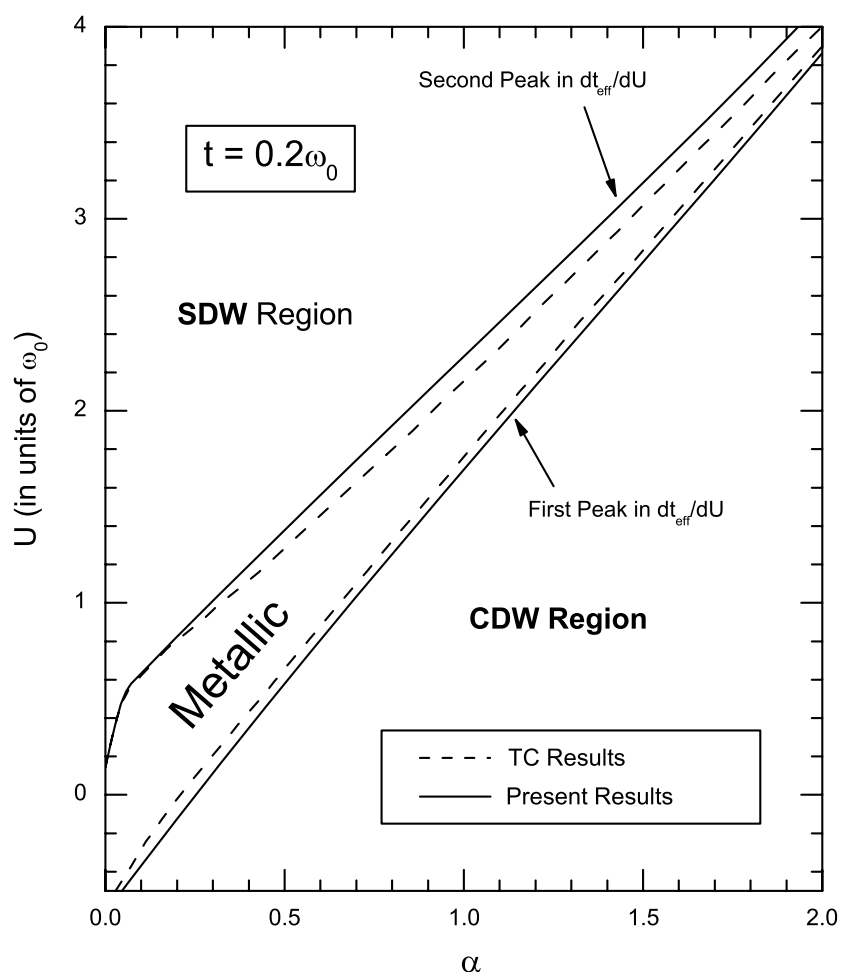

Fig. 3. Phase diagram in $(\alpha, U)$ determined by the peaks in $\mathrm{d} t_{\mathrm{eff}} / \mathrm{d} U$. The corresponding phase diagram obtained in [6] is shown in dashed lines for comparison.

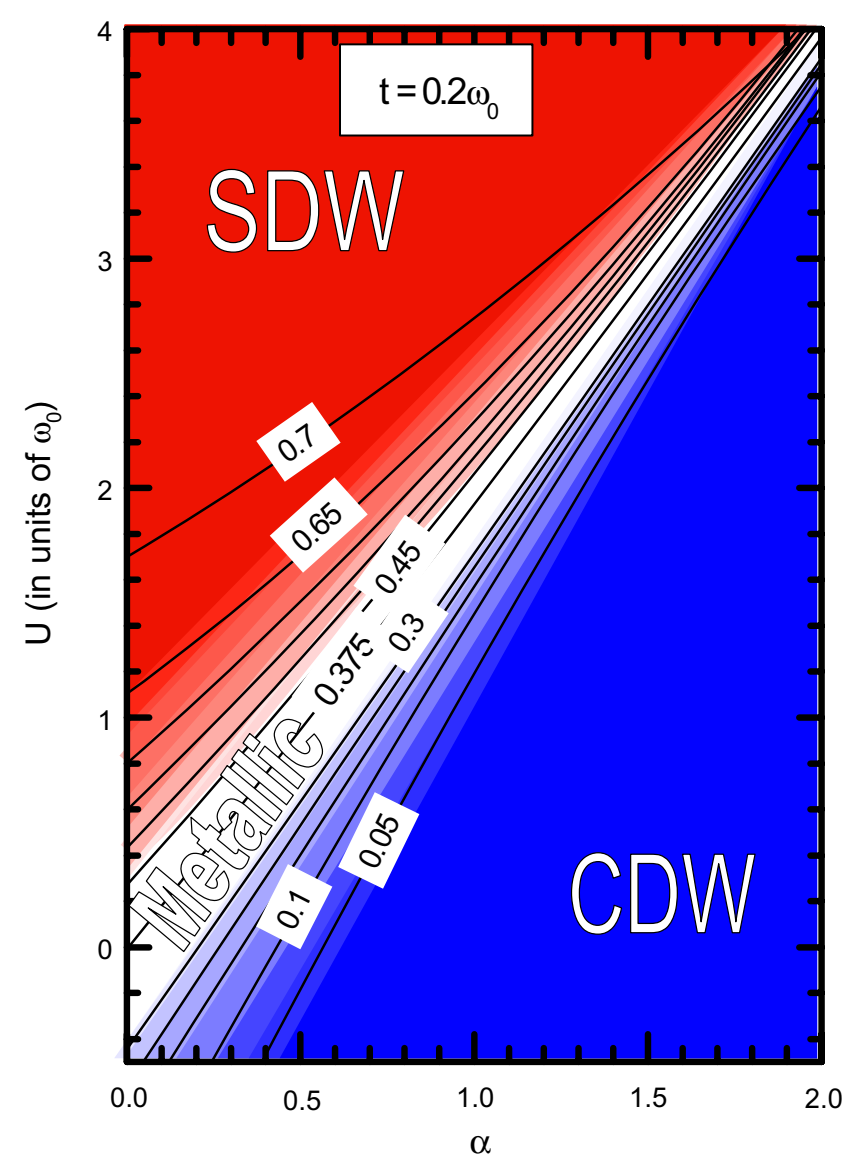

Fig. 4. Contour plots of the local moment $L_{0}$ in $(\alpha, \omega)$ plane.

metallic phase becomes much wider when we use an improved variational calculation.

In conclusion, we have studied the 1D Holstein-Hubbard model at half-filling using a series of canonical transformations to eliminate the phonons in a more accurate way than was done in [13] and have shown that using a better variational calculation widens the intermediate metallic phase at the CDW-SDW crossover region and makes the polarons more mobile. Including the phonon anharmonicity at the hamiltonian level will make the intermediate metallic phase even more wider, as shown in [13], and the polarons more mobile which is certainly a more favourable situation for high- $T_{\mathrm{c}}$ superconductivity. It is of course possible to explore the superconducting nature of this phase within the present scenario. In fact, it goes without saying that being a metallic phase, this phase will certainly be superconductive as one will cool the temperature. However, to find the transition temperature itself one does need to do a rigorous calculation which itself will be the content of a separate investigation. It is however worthwhile to make a few qualitative remarks about the nature of the superconducting phase. The normal phase here is a polaronic or a bipolaronic metal. Therefore, as the temperature would decrease, the system can undergo a polaronic superconductivity induced by dynamical pairing of polarons like what happens in the case of Cooper pairs in the BCS mech- 
anism. On the other hand, it is also possible that bipolarons which are essentially "static" Cooper pairs can undergo Bose-Einstein condensation and give rise to a superconductive phase. Which route will actually lead to the superconductive phase in an actual system will depend on the characteristic temperatures of the corresponding mechanisms which will certainly depend on the material parameters and, therefore, requires a rigorous calculation. This calculation is in progress and will be reported in due course.

\section{Acknowledgements}

R.P.M.K. thanks CSIR, India for financial support. Numerical computations for this work were performed using the CMSD facility of the University of Hyderabad.

\section{References}

[1] A.S. Alexandrov, J. Ranninger, Phys. Rev. B 23 (1981) 1796; A.N. Das, S. Sil, Physica C 161 (1989) 325;

E. Berger, P. Valášek, W. von der Linden, Physica B 52 (1995) 4806; S. Sil, B. Bhattacharyya, Phys. Rev. B 54 (1996) 349;

C.H. Pao, H.B. Schüttler, Phys. Rev. B 57 (1998) 5051;

A. Weiße, H. Fehske, W. Wellein, A.R. Bishop, Phys. Rev. B 62 (2000) R747;
V. Cataudella, G. De Fillipis, G. Iadonisi, Phys. Rev. B 62 (2000) 1496;

W. Koller, A.C. Hewson, D.M. Edwards, Phys. Rev. Lett. 95 (2005) 256401;

G. Sangiovanni, M. Capone, C.C. Castellani, M. Grilli, Phys. Rev. Lett. 94 (2005) 026401;

P. Barone, R. Raimondi, M. Capone, C. Castellani, Phys. Rev. B 73 (2006) 085120.

[2] S. Alexandrov, N.F. Mott, Rep. Prog. Phys. B57 (1994) 1197.

[3] R. Micnas, J. Ranninger, S. Robaszkiewicz, Rev. Mod. Phys. 62 (1990) 113.

[4] A.J. Mills, P.B. Littlewoodand, B.I. Shraiman, Phys. Rev. Lett. 74 (1995) 5144;

K.H. Kim, J.Y. Gu, H.S. Choi, G.W. Park, T.W. Noh, Phys. Rev. Lett. 77 (1996) 1877.

[5] B.K. Chakraverty, D. Feinberg, H. Zheng, M. Avignon, Solid State Commun. 64 (1987) 1147;

D. Emin, Phys. Rev. Lett. 62 (1989) 1544;

A. Chatterjee, S. Sil, Mod. Phys. Lett. B 6 (1992) 959.

[6] Y. Takada, A. Chatterjee, Phys. Rev. B 67 (2003) 081102(R).

[7] R.T. Clay, R.P. Hardikar, Phys. Rev. Lett. 95 (2005) 096401.

[8] I. Lang, Y.A. Firsov, Sov. Phys. JETP 16 (1963) 1301; A.N. Das, S. Sil, J. Phys.: Condens. Matter 5 (1993) 8265.

[9] E.H. Lieb, F.Y. Wu, Phys. Rev. Lett. 20 (1968) 1445.

[10] T.K. Mitra, A. Chatterjee, S. Mukhpadhyay, Phys. Rep. 153 (1987) 91.

[11] H. Zheng, Phys. Lett. A 131 (1988) 115.

[12] C.F. Lo, R. Sollie, Phys. Rev. B 48 (1993) 10183.

[13] A. Chatterjee, Y. Takada, J. Phys. Soc. Jpn. 73 (2004) 964. 